\title{
Fetal and Neonatal Responses to Extended Maternal Canine Starvation. I. Circulating Fuels and Glucose and Lactate Turnover
}

\author{
E-L. MIETTINEN, R. M. KLIEGMAN, ${ }^{(31)}$ AND K-Y TSERNG \\ Division of Pediatric Metabolism, Case Western Reserve University, Cleveland Metropolitan General Hospital, \\ Cleveland, Ohio, USA
}

\section{Summary}

The effect of maternal starvation for 5 days on circulating fuels, on glucose, and on lactate turnover in fasted newborn dogs was studied at $0,3,6,9$, and $24 \mathrm{~h}$ of age. Maternal starvation reduced the birthweight of the puppies by $23 \%(232 \pm 6$ versus $300 \pm 10 \mathrm{~g})$. Fetal ketone body concentration $(3.33 \mu \mathrm{mole} / \mathrm{ml})$ paralleled the marked maternal hyperketonemia $(5.78 \mu \mathrm{mole} / \mathrm{ml})$.

After birth, maternal canine starvation (MCS) also caused significantly lower fasting blood glucose values in the pups at $3 \mathrm{~h}$ of age compared to controls (2.79 versus $5.77 \mu$ mole/ml). In addition, plasma free fatty acids (FFA) and glycerol levels were significantly reduced at $3 \mathrm{~h}$ of age. At $6 \mathrm{~h}$, glucose and FFA concentration became equivalent to controls. Glycerol concentration, however, remained depressed at 6 and $9 \mathrm{~h}$, and plasma triglyceride levels were significantly elevated. A significant rise in blood alanine concentration was observed at 3,6 , and $24 \mathrm{~h}$ of age in MCS pups. In spite of low blood glucose values at $3 \mathrm{~h}$ of age, the glucose production rate (GPR) in the MCS pups was the same as in the controls. Lactate turnover and its carbon incorporation into glucose did not show any significant differences between MCS and control pups at individual time points.

These data suggest that during starvation the mother was unable to provide sufficient substrates for normal fetal growth. Maternal and fetal nutrient deficiency may have resulted also in decreased neonatal blood glucose levels together with reduced lipolysis in the immediate newborn period. Circulating glucose, FFA, and glycerol concentrations reached control values during the remainder of the study. Diminished availability of FFA may have resulted in enhanced tissue clearance of glucose. When lipolysis increased and peripheral tissue had an alternate fuel, glucose clearance returned to control values. The precise mechanism for the diminished levels of circulating fuels among fasted pups after 5 days of maternal starvation was not directly evident from these investigations of circulating fuels and glucose or lactate turnover data.

\section{Abbreviation}

FFA, free fatty acids

GPR, glucose production rate

MCS, maternal canine starvation

Maternal starvation has been shown to reduce fetal birthweight and cause a significant decrease in fetal blood glucose concentration and an increase in fetal ketone body levels in different mammalian species $(1,7,9,23)$.

The effect of maternal starvation upon fasting neonatal metabolic adaptation to birth has been investigated in dogs after 3 days of maternal starvation $(12,15)$. In these earlier studies maternal starvation caused a very small fetal weight loss and an increase in fetal blood ketone body levels. During the early hours of neonatal life, pups of these starved mothers had decreased fasting blood glucose and plasma FFA levels; at the same time glucose production rates were also attenuated. It was hypothesized that limited hepatic FFA oxidation may have delayed the activation of gluconeogenesis needed to enhance hepatic glucose production.

As 3 days of maternal canine starvation had only a small effect on intrauterine fetal growth, the present investigation reports observations with a more extended maternal fasting period. This extended maternal canine starvation markedly reduced fetal growth and resulted in circulating substrate profiles that are similar to those observed in hypoglycemic human infants with severe intrauterine growth retardation (14). The fatty acid-glucose interrelationship, hypothesized to be essential for neonatal hepatic gluconeogenesis and spare peripheral tissue glucose utilization, was examined in this extended model which resulted in marked intrauterine growth retardation.

\section{MATERIALS AND METHODS}

D- $\left[6-{ }^{3} \mathrm{H}\right]$ Glucose and $\mathrm{L}-\left[\mathrm{U}-{ }^{14} \mathrm{C}\right]$ lactate were purchased from New England Nuclear, Boston, MA. All enzymes were purchased from Sigma, St. Louis, MO, except $\mathrm{L}$-glutamate dehydrogenase which was purchased from Cal-Biochem, La Jolla, CA.

Animals. Pregnant beagle dogs were maintained in kennels and fed a standard canine diet. Before delivery, six mothers were fasted overnight $(18 \mathrm{~h}$ ) providing a control group, while eight were fasted for 5 days providing the MCS groups. Cesarean section was done at term ( $60 \pm 2$ days). The newborn pups were immediately transferred to an incubator maintained at $37^{\circ} \mathrm{C}$ with relative humidity of $70 \%$. The newborn puppies were fasted throughout the study period in both groups. Litter size in the control group was between $3-8(5.3 \pm 0.5$, mean \pm S.E.M. $)$ and between 1-7 $(4.6 \pm 0.8)$ in the MCS group.

The number of pups analyzed may alter at different times because we were not always able to obtain sufficient quantities of blood for every analysis from each mother and pup. The effect of maternal starvation on intrahepatic intermediates was also evaluated. The results are reported in accompanying paper (16).

Experimental design. Before Cesarean section, intravenous anesthesia ( $4 \mathrm{mg} / \mathrm{kg}$ chlorpromazine) was given to the mother. Although thorazine crosses the placenta, values obtained in humans are much lower in the fetus and do not affect clinical behavior (17). Furthermore usual doses of thorazine have minimal or no effect on canine cerebral oxygen consumption and do not alter cerebral blood flow (22). Nonetheless, our study compares two groups treated with identical perinatal anesthesia. Any possible small effect should not confuse the comparison of MCS and control pups treated in the same manner. Lidocaine (2\%) was used to anesthesize the incision area. Paired blood samples were taken from the mother and, immediately after delivery, from one or two fetuses. We realize that these values represent maternal and fetal substrates at the time of delivery and may not be quantitatively related to substrate levels in the unanesthetized and unhandled resting state. The newborn pups were permitted to stabilize, were 
weighed, and had umbilical arterial and venous catheters ( $3.5 \mathrm{Fr}$. Argyle, St. Louis, MO) placed atraumatically. Pups from each litter were randomly selected for infusion lasting $3 \mathrm{~h}$, so that the infusion ended at $3,6,9$, and $24 \mathrm{~h}$ of age. The pups were infused with trace quantities of $\left[6{ }^{3} \mathrm{H}\right] \mathrm{glucose}(\mathrm{SA} 33.9 \mathrm{Ci} / \mathrm{mmole}$ ) and [U- ${ }^{14} \mathrm{C}$ ]lactate (SA $138 \mathrm{mCi} / \mathrm{mmole}$ ) dissolved in sterile isotonic saline as described before $(11,12)$.

Analyses. Blood glucose, lactate and their specific activities, amino acids, triglyceride, and ketone levels were measured as described earlier (11). FFA were analyzed using the gas chromatographic method of Tserng et al. (26), plasma glycerol levels were measured by modified glycerokinase method by Centrifichem (2). Glucose production rate and lactate turnover were calculated from tracer dilution according to Steele's equation (24). The lactate carbon incorporation into glucose was calculated by comparing the $\left[{ }^{14} \mathrm{C}\right]$ specific activity of glucose and lactate given the limitations of this method as a semi-quantitative technique for determining gluconeogenesis, discussed in detail previously (12).

Statistics. All results were expressed as mean \pm S.E.M. The differences between groups were determined by the Student's $t$ test.

\section{RESULTS}

Five days of maternal starvation caused a significant weight loss at birth among the pups studied. Average puppy weight of 32 controls was $300 \pm 10 \mathrm{~g}$. It was $232 \pm 6 \mathrm{~g}$ in the 37 offspring of starved mothers $(P<0.0001)$. No difference in clinical behavior of the puppies in both groups was observed. All studied pups were pink in room air, active, and fully conscious and mobile during the study. An identical group of pups had normal oxygen consumption, respiratory quotients and arterial oxygen saturations (13).

\section{CIRCULATING FUELS}

Glucose and lactate (Table 1). Even though fasting for 5 days lowered the maternal blood glucose concentration significantly, fetal blood glucose levels appeared lower but were not significantly reduced as compared with the controls. After birth, blood glucose concentration rose in control pups during the first $3 \mathrm{~h}$ and remained constant throughout the first day. In contrast, blood glucose levels in the MCS group remained low up to $3 \mathrm{~h}$ of age, being significantly lower than in the controls at this time and then reaching the control levels during the remainder of the study.

Maternal lactate concentrations did not show any effects of starvation. Fetal lactate levels were elevated compared to mothers in both groups studied. By $3 \mathrm{~h}$ of age, lactate levels declined in both groups; however, in the MCS group (compared to timed control pups) the concentrations were elevated at 6 and $9 \mathrm{~h}$, the elevation being significant only at $6 \mathrm{~h}$.

Plasma FFA, glycerol, triglycerides, and ketone bodies (Fig. 1). Maternal starvation did not effect maternal or fetal plasma FFA concentrations. After birth, control pup plasma FFA values rose above their respective fetal levels. In the MCS pups, however, plasma FFA concentration was significantly lower than in controls at $3 \mathrm{~h}$ of age. Plasma glycerol concentrations also rose after birth in both groups, but were significantly lower in the MCS pups at 3,6 , and $9 \mathrm{~h}$ of age. Plasma triglycerides increased after birth and reached higher than fetal levels in both groups studied. Compared with timed controls, plasma triglycerides in MCS pups were significantly elevated at 6 and $9 \mathrm{~h}$. At $24 \mathrm{~h}$, triglyceride concentrations markedly declined in both groups studied.

Maternal starvation for 5 days caused a marked maternal and fetal ketosis. By the age of $3 \mathrm{~h}$, plasma ketones in the MCS pups declined to within the control range. Ketone body concentrations then remained unchanged during $24 \mathrm{~h}$ of neonatal fasting.

Blood amino acids (Table 2). Of the gluconeogenic amino acids studied, only glutamate demonstrated a significant decrease in the mother after 5 days of starvation. As expected, alanine, glutamate, and glutamine concentrations were higher in all the fetus-pups than in their mothers and there were no differences between the two pup groups. After birth, alanine, glutamate, and glutamine concentrations decreased in all pups. Compared with controls, alanine levels remained significantly elevated at 3,6 , and $24 \mathrm{~h}$ of age. Glutamate levels were lower in the MCS pups only at $3 \mathrm{~h}$ of age whereas glutamine concentrations were lower only at $9 \mathrm{~h}$.

Neonatal glucose production rate and lactate turnover (Table 3). A steady state for glucose and lactate specific activities and concentrations was achieved in all pups investigated. Under these conditions glucose production equals glucose utilization. In the control group, glucose production rates did not show any significant changes throughout the $24 \mathrm{~h}$. In the MCS group, there was an increase in glucose production rates from $3 \mathrm{~h}$ to the end of the study. At $24 \mathrm{~h}$, glucose turnover in the MCS pups became significantly higher than that in the controls.

At $3 \mathrm{~h}$ of age blood glucose concentrations were depressed whereas glucose production and utilization were unaffected. Glucose clearance among MCS pups became significantly elevated at this time period. Clearance was also elevated at $24 \mathrm{~h}$ of age.

Lactate turnover remained unchanged in all the pups studied during the first day of life, and there were no differences between the two groups studied. Lactate carbon incorporation into glucose increased significantly $(P<0.05)$ in both study groups over the 24-h period. Incorporation of lactate into glucose appeared higher in the MCS group, but because of large variance the differences never achieved statistical significance.

\section{DISCUSSION}

In contrast to 3 days of MCS, starvation for 5 days in dogs caused a significant decline in the maternal blood glucose concentration. Felig and Lynch (5) have also demonstrated continuous decrease in maternal blood glucose concentration up to $84 \mathrm{~h}$ of starvation in pregnant humans in early gestation. Because of increased maternal FFA mobilization and subsequent oxidation, markedly elevated plasma ketone body levels were also observed after 5 days of maternal canine starvation. Low maternal blood

Table 1. Blood glucose and lactate concentrations after extended material canine starvation ( $\mu$ mole $/ m l$, mean \pm S.E.M.)

\begin{tabular}{|c|c|c|c|c|c|c|}
\hline & \multicolumn{2}{|c|}{ At birth } & \multicolumn{4}{|c|}{ Newborn dog } \\
\hline & Mother & Fetus & $3 \mathrm{~h}$ & $6 \mathrm{~h}$ & $9 \mathrm{~h}$ & $24 \mathrm{~h}$ \\
\hline \multicolumn{7}{|l|}{ Glucose } \\
\hline Control & $4.14 \pm 0.251$ & $3.64 \pm 0.198$ & $5.77 \pm 1.11$ & $4.84 \pm 0.737$ & $5.24 \pm 0.776$ & $4.65 \pm 0.567$ \\
\hline $\mathrm{MCS}^{1}$ & $3.22 \pm 0.432^{2}$ & $2.83 \pm 0.486$ & $2.79 \pm 0.452^{2}$ & $4.05 \pm 0.847$ & $6.74 \pm 1.56$ & $3.24 \pm 0.814$ \\
\hline \multicolumn{7}{|l|}{ Lactate } \\
\hline Control & $1.39 \pm 0.254$ & $3.23 \pm 0.213$ & $1.76 \pm 0.283$ & $1.52 \pm 0.211$ & $1.64 \pm 0.192$ & $1.67 \pm 0.226$ \\
\hline MCS & $1.51 \pm 0.159$ & $3.98 \pm 0.532$ & $1.72 \pm 0.238$ & $2.17 \pm 0.304^{2}$ & $2.15 \pm 0.351$ & $1.81 \pm 0.301$ \\
\hline \multicolumn{7}{|c|}{ Number studied } \\
\hline Control & 6 & 5 & 6 & 6 & 6 & 6 \\
\hline MCS & 7 & 7 & 9 & 8 & 5 & 6 \\
\hline
\end{tabular}

${ }^{1}$ MCS, prior maternal canine starvation.

${ }^{2} P<0.05$. 


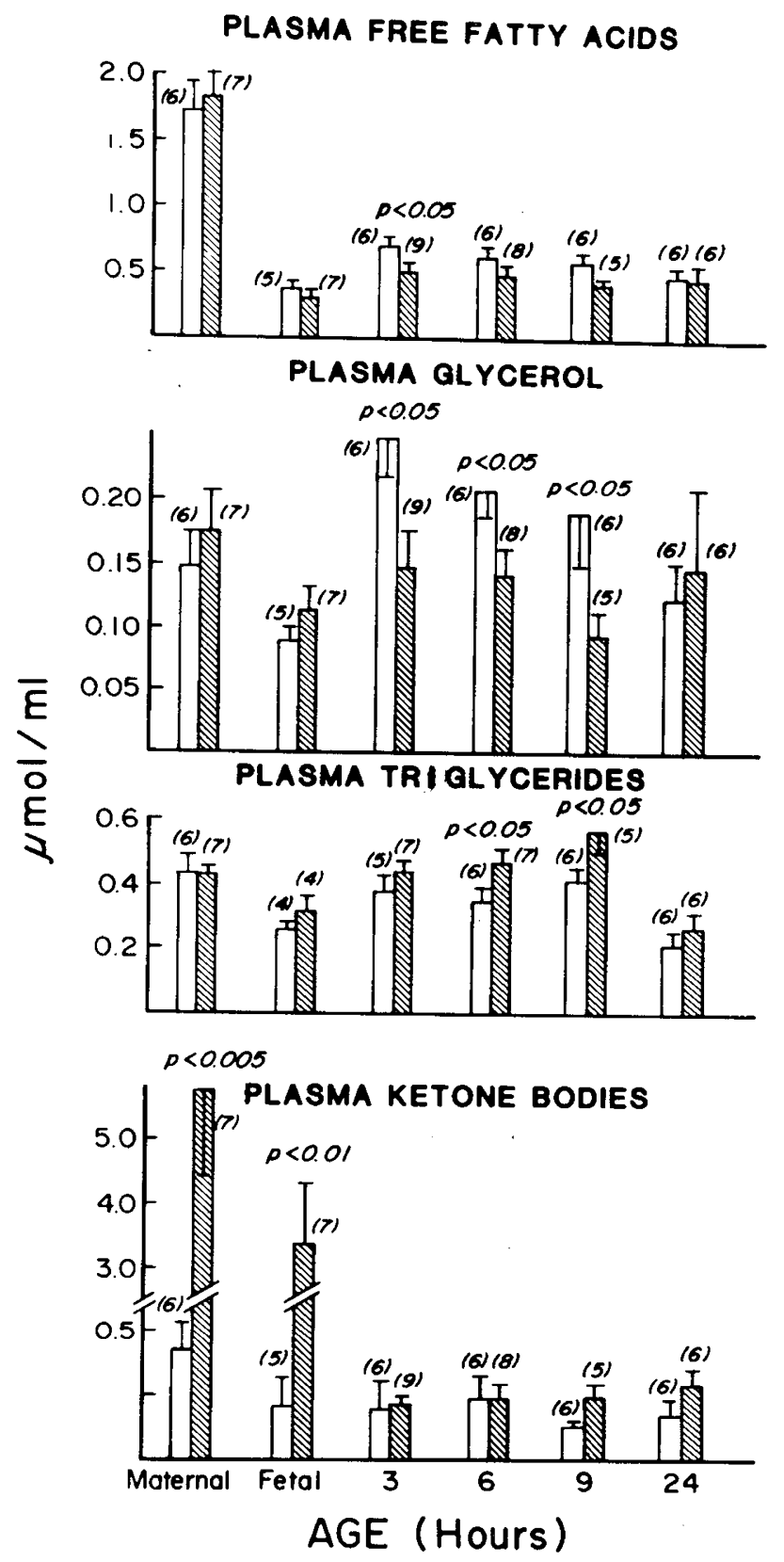

Fig. 1. Maternal, fetal, and neonatal plasma free fatty acid, glycerol, triglyceride, and ketone body concentrations (mean \pm S.E.M.). Control pups $\square$; prior extended maternal starvation and number of pups (W).

glucose and high plasma ketone body concentrations together with a significant (23\%) weight loss in the pups, facilitates a better evaluation of the effects of altered substrate provision to the fetus and its effects on fasting neonatal metabolism compared with the shorter 3-day period of maternal canine starvation (12).

Elevated fetal plasma ketone body levels and an unaltered fetal blood glucose concentration at the time of birth suggest that these fetuses received alternate fuels from the mother during the extended period of maternal starvation; nevertheless, the marked reduction of birthweight suggests that net fuel provision and/or utilization needed to be diminished. After birth, fasted MCS pups developed significant alterations in circulatory fuels. In particular, pups of the starved mothers were not able to increase their blood glucose concentrations at $3 \mathrm{~h}$ of age as did the control pups. This was also observed previously after 3 days of maternal starvation (12).

In the present investigation, fasting neonatal glucose production was not altered during the initial 3-h period after birth when blood glucose concentrations were lower in MCS pups. As blood glucose levels and specific activity were constant, (in a steady state) glucose utilization was also unaltered despite the lower blood glucose levels, necessitating that systemic rates of glucose clearance were enhanced. As this metabolic profile (diminished blood glucose and enhanced glucose clearance) was only demonstrated immediately after birth, the events related to fetal starvation and the immediate transition to fasting neonatal metabolism may have been most severely affected by MCS.

Maternal ovine starvation has been demonstrated to reduce fetal glucose availability and umbilical glucose uptake, producing fetal hypoglycemia (20). Maternal canine starvation may have resulted in a similar sequence of fetal metabolic events. The postnatal response to prior diminished fetal glucose uptake may result in enhanced tissue glucose extraction (clearance) and neonatal hypoglycemia despite apparent unaltered rates of neonatal systemic glucose production. In the presence of attenuated circulating levels of alternate fuels, such as FFA and glycerol, systemic oxidative metabolism may be predominantly supported by glucose in the MCS pups.

As FFA should spare systemic glucose oxidation under normal circumstances, the presence of diminished plasma FFA and glycerol levels suggest that excessive glucose must obligatively be extracted or cleared from the circulation to maintain tissue energy production (6). As both FFA and glycerol levels were diminished at $3 \mathrm{~h}$, it is most likely that lipolysis was altered in the immediate transition to birth after MCS rather than enhanced tissue utilization of these two substrates.

After this period ( $3 \mathrm{~h}$ of age) of hypoglycemia, the MCS pups adapted to neonatal fasting and demonstrated blood glucose concentrations that became equivalent to controls. In addition, at 3 , 6 , and $9 \mathrm{~h}$ of age glucose turnover was similar in both pup groups; nevertheless, the absolute rate of glucose production increased during the day only within the MCS pups and eventually became higher than that in the time controlled normal pups at $24 \mathrm{~h}$ of neonatal fasting. As the absolute decline of hepatic glycogen content was equivalent in both groups during the 24-h fasting period, the contribution of gluconeogenesis to this apparent increase of neonatal glucose turnover within the MCS pups (between 3-24 h) may become important $(10,16)$. Again, when comparing the rate of lactate carbon incorporation into glucose within the MCS pups alone, one notes a very significant increase during the 24-h study period. Although rates of the estimation of gluconeogenesis during this time period only doubled within control pups, lactate carbon incorporation into glucose increased over 4-fold and should contribute to a greater proportion of hepatic glucose output, as neonatal fasting was continued to $24 \mathrm{~h}$ of age. Nonetheless, caution should be made in interpretation of lactate carbon incorporation into glucose as it may only represent glucose recycling rather than net gluconeogenesis. During fasting and/or periods of enhanced hepatic glycolytic flux, recycling may increase $(11,15)$.

The elevated fetal ketone bodies were cleared within $3 \mathrm{~h}$ by both newborn groups. Ketones may be utilized by the fetus and neonate as alternate oxidative fuels in systemic tissue $(20,21)$ or for lipogenesis by others $(18,19)$. Adipose tissue lipolysis may be attenuated, as discussed above (27), in view of the absence of the marked elevation of plasma FFA and glycerol levels after birth among MCS pups. Alternatively, low plasma FFA and glycerol levels may be due to increased hepatic uptake or re-esterification into triglycerides (4). The elevated plasma triglyceride levels in MCS pups, observed at 6 and $9 \mathrm{~h}$ of age, are probably due to diminished hepatic fatty acid oxidation resulting in enhanced hepatic triglyceride esterification. Alternately, diminished fat utilization by peripheral tissue may also result in higher plasma triglyceride levels in MCS pups. Similar observations of diminished FFA and subsequently elevated triglycerides were observed in MCS pups after 3 days of maternal starvation (12). Neonatal hepatic intermediate data from both 3-day and 5-day periods of maternal starvation also suggest that intrahepatic fatty acid oxidation is attenuated during neonatal fasting among MCS pups $(15,16)$.

It has also been demonstrated that amino acids may function as 
Table 2. Blood amino acid concentrations after extended maternal canine starvation ( $\mu$ mole/ml, mean \pm S.E.M.)

\begin{tabular}{|c|c|c|c|c|c|c|}
\hline & \multicolumn{2}{|c|}{ At birth } & \multicolumn{4}{|c|}{ Newborn dog } \\
\hline & Mother & Fetus & $3 \mathrm{~h}$ & $6 \mathrm{~h}$ & $9 \mathrm{~h}$ & $24 \mathrm{~h}$ \\
\hline \multicolumn{7}{|l|}{ Alanine } \\
\hline Control & $0.240 \pm 0.042$ & $0.345 \pm 0.042$ & $0.140 \pm 0.021$ & $0.106 \pm 0.016$ & $0.114 \pm 0.011$ & $0.139 \pm 0.031$ \\
\hline $\mathrm{MCS}^{1}$ & $0.199 \pm 0.027$ & $0.421 \pm 0.064$ & $0.237 \pm 0.029^{2}$ & $0.220 \pm 0.022^{3}$ & $0.191 \pm 0.043$ & $0.250 \pm 0.028^{4}$ \\
\hline \multicolumn{7}{|l|}{ Aspartate } \\
\hline Control & $0.082 \pm 0.011$ & $0.095 \pm 0.014$ & $0.089 \pm 0.015$ & $0.082 \pm 0.009$ & $0.074 \pm 0.006$ & $0.088 \pm 0.012$ \\
\hline MCS & $0.075 \pm 0.006$ & $0.082 \pm 0.004$ & $0.077 \pm 0.007$ & $0.076 \pm 0.010$ & $0.075 \pm 0.011$ & $0.073 \pm 0.012$ \\
\hline \multicolumn{7}{|l|}{ Glutamate } \\
\hline Control & $0.096 \pm 0.009$ & $0.144 \pm 0.026$ & $0.107 \pm 0.016$ & $0.105 \pm 0.025$ & $0.107 \pm 0.018$ & $0.105 \pm 0.036$ \\
\hline MCS & $0.059 \pm 0.013^{4}$ & $0.164 \pm 0.034$ & $0.069 \pm 0.009^{4}$ & $0.085 \pm 0.012$ & $0.092 \pm 0.016$ & $0.083 \pm 0.015$ \\
\hline \multicolumn{7}{|l|}{ Glutamine } \\
\hline Control & $0.648 \pm 0.024$ & $1.105 \pm 0.132$ & $0.789 \pm 0.139$ & $0.597 \pm 0.035$ & $0.582 \pm 0.033$ & $0.505 \pm 0.052$ \\
\hline MCS & $0.716 \pm 0.239$ & $0.954 \pm 0.103$ & $0.680 \pm 0.045$ & $0.675 \pm 0.163$ & $0.495 \pm 0.030^{4}$ & $0.591 \pm 0.019$ \\
\hline \multicolumn{7}{|c|}{ Number studied } \\
\hline Control & 6 & 5 & 6 & 6 & 6 & 6 \\
\hline MCS & 7 & 7 & 9 & 8 & 5 & 5 \\
\hline
\end{tabular}

${ }^{1}$ MCS, prior maternal canine starvation.

${ }^{2} P<0.01$.

${ }^{3} P<0.001$.

${ }^{4} P<0.05$.

Table 3. Glucose and lactate turnover and lactate carbon incorporation into glucose after extended maternal canine starvation ( $\mu$ mole/ $(\mathrm{kg} \cdot \mathrm{min})$, mean \pm S.E.M.)

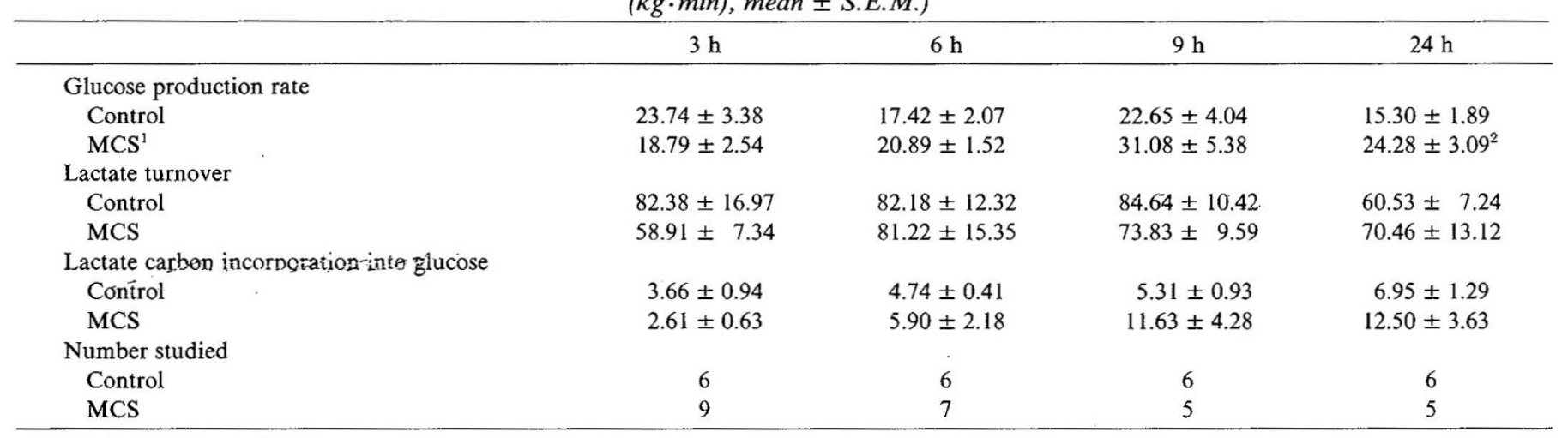

${ }^{1}$ MCS, prior maternal canine starvation.

${ }^{2} P<0.05$.

alternate fuels for fetal and newborn animals as these fuels may either be directly oxidized or serve as precursors for gluconeogenesis $(3,18,23,28)$. From the amino acids studied in these pups, circulating alanine, glutamate, and glutamine concentrations were higher in the fetus than in the mother as shown in earlier studies (25). After birth, these three amino acid levels decreased in the neonatal circulation whereas alanine (and lactate, another gluconeogenic precursor) concentrations were higher in the MCS pups than in the controls during the first day of life. Alanine and lactate have also been shown to be increased among human infants with intrauterine growth retardation (14). This may possibly be due to increased alanine release from the muscle and/or limited hepatic uptake and utilization for gluconeogenesis (14). Lack of an early induction of gluconeogenesis during the period of hypoglycemia among the MCS pups may be due to decreased hepatic FFA oxidation (6), which may provide energy and the necessary reducing equivalents needed for hepatic gluconeogenesis as demonstrated in other mammalian species (29).

Maternal canine starvation for five days produced a significant fetal weight loss, lowered immediate neonatal blood glucose concentrations despite normal glucose production rates, and decreased plasma FFA and glycerol availability at three hours of age. In addition, plasma triglycerides were either normal or elevated while alanine levels were higher in MCS pups throughout the first day. This animal model is more representative of the circulating biochemical alterations noted among intrauterine growth retarded infants, and is more suitable for study than the less prolonged three days of MCS $(12,14)$. To be able to further understand the mechanisms for diminished circulating fasting glucose and FFA levels, important hepatic intermediates and energy metabolites from these same dogs were analyzed and are reported in the companion paper (16).

\section{REFERENCES AND NOTES}

1. Boyd, R. D., Morris, F. H., Meschia, G., Makowski, E. L., and Battaglia, F. C.: Growth of glucose and oxygen uptakes by fetuses of fed and starved ewes. Am. J. Physiol., 225: 897 (1973).

2. Bucolo, G. and David, H.: Quantitative determination of serum triglycerides by the use of enzymes. Clin. Chem., 19: 476 (1973).

3. Cresteil, T. and LeRoux, J-P.: Early postnatal metabolism of amino acids in rat. Pediatr. Res., 11: 720 (1977).

4. Elphick, M. C., Edson, J. L., Lawcor, J. P., and Hull, D.: Source of fetal-stored lipids during maternal starvation in rabbits. Biol. Neonate, 34: 146 (1978).

5. Felig, P. and Lynch, W.: Starvation in human pregnancy: hypoglycemia, hypoinsulinemia and hyperketonemia. Science, 170: 990 (1970).

6. Ferre, P., Pegorier, J. P., Marliss, E. B., and Girard, J. R.: Influence of exogenous fat and gluconeogenic substrates on glucose homeostasis in the newborn rat. Am. J. Physiol. (Endocrinol. Metab. Gastrointest. Physiol. 3), 234: E129 (1978).

7. Girard, J. R., Ferre, P., Gilbert, M., Kervran, A., Assan, R., and Marlis, E. B Fetal metabolic response to maternal fasting in the rat. Am. J. Physiol (Endocrinol. Metab. Gastrointest. Physiol. 1), 232: E456 (1977).

8. Girard, J. R., Guillet, I., Marty, J., and Marliss, E. B.: Plasma amino acid levels and development of hepatic gluconeogenesis in the newborn rat. Am. J. Physiol., 229(2): 466 (1975). 
9. Goodner, C. J. and Thompson, D. J.: Glucose metabolism in the fetus in utero: The effect of maternal fasting and glucose loading in the rat. Pediatr. Res., 1 . 443 (1967)

10. Kawai, Y. and Arinze, J.: Activation of glycogenolysis in neonatal liver. J. Biol Chem., 256: 853 (1981).

11. Kliegman, R. M., Miettinen, E-L., and Adam, P. A. J.: Substrate turnover interrelationships in fasting neonatal dogs. Am. J. Physiol. (Endocrinol. Metab. 2), 239: E287 (1980).

12. Kliegman, R. M., Miettinen, E-L., and Adam, P. A. J.: Fetal and neonatal responses to maternal canine starvation: circulating fuels and neonatal glucose production. Pediatr. Res., 15: 945 (1981).

13. Kliegman, R. M., Miettinen, E. L., Campbell G., and Patel, M.: Fasting neonatal canine oxidative metabolism following maternal starvation. Pediatr. Res. (abstract), 16: 113A (1982).

14. Mestyan, J., Soltesz, G., Schultz, K., and Horwath, M.: Hyperaminoacidemia due to the accumulation of gluconeogenic amino acid precursors in hypoglycemic small-for-gestational age infants. J. Pediatr., 87: 409 (1975).

15. Miettinen, E-L.: Effect of maternal canine starvation on fetal and neonatal liver metabolism. Am. J. Physiol. (Endocrinol. Metab. 3), 240: E88 (1981).

16. Miettinen, E-L. and Kliegman, R. M.: Fetal and neonatal responses to extended maternal starvation. II. Fetal and neonatal liver metabolism. Pediatr. Res. 17: 639 (1983).

17. Nahas, G. and Goujard, J.: Phenothiazines, benzodiazepines and the fetus. Rev Perinatol. Med., 3: 243 (1979).

18. Seccombe, D. W., Harding, P. G. R., and Possmayer, F.: Fetal utilization of maternally derived ketone bodies for lipogenesis in the rat. Biochem. Biophys. Acta, 488: 402 (1977).

19. Shambaugh, G. E.: A potential lipid source for fetal liver. Am. J. Clin. Nutr. (abstract), 33: 945 (1980).

20. Shambaugh, G. E., Koehler, R. A., and Freinkel, N.: Fetal fuels. II. Contributors of selected carbon fuels to oxidative metabolism in rat conceptus. Am. J. Physiol. (Endocrinol. Metab. Gastrointest. Physiol. 2), 233: E457 (1977).

21. Shambaugh, G. E., Koehler, R. A., and Yokoo, H.: Fetal Fuels III. Ketone utilization by fetal hepatocyte. Am. J. Physiol. (Endocrinol. Metab. Gastrointest. Physiol. 4), 235: E330 (1978).

22. Siesjo, B.: Brain energy metabolism. (John Wiley \& Son, New York, New York, 1978).

23. Simmons, M. A., Meschia, G., Makowski, E. L., and Battaglia, F. C.: Fetal metabolic response to maternal starvation. Pediatr. Res., 8: 830 (1974)

24. Steele, R., Wall, J. S., De-Bodo, R. C., and Altszuler, N.: Measurement of size and turnover rate of body glucose pool by isotope dilution method. Am. J. Physiol., 187: 15 (1956).

25. Stegink, L. D., Pitkin, R. M., Reynolds, W. A., Brummel, M. C., and Filer, L. J. Jr.: Placental transfer of aspartate and its metabolites in the primate. Metabolism, 28: 669 (1979).

26. Tserng, K-Y., Kliegman, R., Miettinen, E-L., and Kalhan, S.: A rapid, simple and sensitive procedure for the determination of free fatty acids in plasma using glass capillary column gas chromatography. J. Lipid Res., 22: 852 (1981)

27. Warshaw, J. B.: Fatty acid metabolism during development. Semin. in Perinatol., 3: 131 (1979).

28. White, P. K. and Miller, S. A.: Utilization of dietary amino acids for energy production in neonatal rat liver. Pediatr. Res., 10: 158 (1976).

29. Williamson, D. H., Lund, P., and Krebs, H. A.: The redox state of free nicotinamide-adenine dinucleotide in the cytoplasm and mitochondria of rat liver Biochem. J., 103: 514 (1967).

30. The authors would like to express their appreciation to Mr. George Campbell, Ms. Sharon McBee, and Ms. Alice Sutton for their technical assistance, and Ms. Sandra Hartman for her professional preparation of this text. We also express our gratitude to Dr. Sherman D. Sachs of Veterinary Medicine of Case

Western Reserve University for surgical and general care of the dogs.
31. Requests for reprints should be addressed to: Dr. R. M. Kliegman, Department of Pediatrics, Rainbow Babies and Childrens Hospital, 2101 Adelbert Road, Cleveland, Ohio 44106

32. This work was supported by HD-05740 from the NICHHD

33. Received for publication February 8,1982

34. Accepted for publication December 13, 1982. 